\title{
STABILITY OF AN EVAPORATING THIN POLYMER FILM
}

\author{
Michael Shusser and Oleg V. Gendelman \\ Faculty of Mechanical Engineering, Technion - Israel Institute of Technology, \\ Haifa 32000, Israel
}

\begin{abstract}
Mesoscopic patterns have been observed to form in thin polymer films undergoing a fast dip-coating process. Though several mechanisms for creating of mesoscopic patterns have been proposed, the mechanism of the evaporation-induced mesoscopic self-organization is not yet fully understood. The paper aims to explain this phenomenon by studying the stability of a thin evaporating film. The analysis does not assume long wave disturbances or quasi-steady base solution. It is found that under these conditions there exists a purely thermal instability mechanism that could be responsible for mesoscopic pattern formation. The instability is caused by increasing of evaporation with the film thinning due to higher heat flux from the substrate to the film surface.
\end{abstract}

Keywords: Polymer, Thin Film, Mesoscopic Pattern, Stability

Nomenclature

A - perturbation amplitude of film thickness

$\mathrm{c}_{\mathrm{p}}$ - specific heat

$\mathrm{h}$ - heat transfer coefficient

$\mathrm{J}$ - evaporation rate

$\mathrm{K}$ - Kutateladze number

$\mathrm{k}$ - heat conductivity

$\mathrm{L}$ - latent heat of evaporation

$\mathrm{s}$ - dimensionless coordinate - equation (25)

$\mathrm{T}$ - temperature

$\mathrm{T}_{\mathrm{S}}$ - saturation temperature

$\mathrm{T}_{\infty}$ - gas temperature

$\mathrm{t}$ - time

$\mathrm{t}_{\mathrm{c}}$ - characteristic time

$\mathrm{x}$ - coordinate along the film

$y$ - coordinate across the film

$\mathrm{z}$ - dimensionless coordinate - equation (25)

$\alpha-$ thermal diffusivity

$\delta$ - film thickness

$\delta_{0}-$ initial film thickness

$\theta^{\prime}$ - dimensionless temperature perturbation - equation (22)

$\hat{\theta}$ - temperature perturbation amplitude

$\kappa$ - perturbation wave number

$\rho$ - density

$\tau$ - dimensionless time - equation (25)

Embellishments

- base value

' - perturbation 


\section{Introduction}

Micrometric polymer structures have attracted considerable interest owing to their importance for various optical applications [1-2]. Recently Bormashenko et al. [3] developed a new method of formation of mesoscopically patterned thin polymer films through a fast dip-coating process. Drying of the polymer solution falling vertically along a metal, glass or polymer substrate resulted in creating of $30-50 \mu \mathrm{m}$ cells within the film. The characteristic size of the cells was practically insensitive to the kind of polymer, solvent or substrate and to the atmospheric humidity but depended strongly on the substrate thickness. The mesocopic pattern was obtained only for thin substrates with a thickness of less then $150 \mu \mathrm{m}$.

So far several mechanisms for creating of mesocopic patterns have been proposed including Maragoni instability [4] and surface-tension-driven instability caused by a plume of solvent-rich fluid [5]. However, the mechanism of the evaporation-induced mesoscopic self-organization is not yet fully understood. The goal of the present work is to demonstrate the existence of a purely thermal instability mechanism that can be responsible for the mesoscopic pattern creation.

Stability of a thin evaporating film is a challenging problem due to the coupling between hydrodynamics, heat transfer and possibly mass diffusion and due to its being inherently unsteady [6]. Typical approaches applied to this problem included the normal mode analysis (quasi-steady base state) [7], the integral method [8] and the lubrication theory (long-wave disturbances) [9]. While the first two approaches are by their nature approximate the latter method which is based on the assumption that the disturbance wave length is much large that the mean thickness of the film was successfully used to study the nonlinear stability of evaporating and condensing liquid films [9]. On the other hand, the typical disturbance wave length in the experiments of Bormashenko et al. [3] should be about the characteristic size of the mesoscopic sell, i.e. $30-50 \mu \mathrm{m}$. Such a wave length is too short for the longwave analysis.

In this work we consider an evaporating thin polymer film studied experimentally in [3] and analyze its stability without the assumption of long wave disturbances. We will see that under these conditions there exists a purely thermal instability mechanism.

\section{Physical considerations and basic state}

The schematics of the dip-coating process used by Bormashenko et al. [3] to produce thin polymer films is shown in Fig. 1. The substrate is pulled vertically from the polymer solution. The liquid film falls along the substrate and solidifies during the evaporation of the solvent.

We now proceed to develop a simplified model of polymer film evaporation in this process.

Viscosity of concentrated polymer solutions is very high [10] and the velocities encountered in the experiments of [3] are therefore low. Under these conditions it is reasonable to neglect the flow velocity altogether. The evaporation rate can be determined by the heat flux to the film surface or by the diffusion of the solvent through the polymer film. The observation of Ref. [3] that the creation of the mesoscopic structures depends very strongly on the substrate thickness suggests that it is the heat flux through the substrate which is important.

We will therefore concentrate on the thermal problem and consider a polymer film with the time-dependent thickness $\delta(t)$ which is heated from above by a gas flow 
with the temperature $T_{\infty}$ and the heat transfer coefficient $h$ and also from below by a heat flux through the substrate (see Fig. 2).

While this heat transfer problem is unsteady, its characteristic time can be estimated as $\mathrm{t}_{\mathrm{c}}=\delta_{0}^{2} / \alpha$, where $\delta_{0}$ is the initial thickness of the film and $\alpha$ is the thermal diffusivity of the polymer solution. For concentrated solutions good estimation of $\alpha$ can be obtained from thermal diffusivity of polymer melts which is about $10^{-7} \mathrm{~m}^{2} / \mathrm{s}$ (see Ref. [10], Table 17.1). Hence for a $50 \mu \mathrm{m}$ film, $\mathrm{t}_{\mathrm{c}}=0.025 \mathrm{~s}$, which is very small in comparison with the film drying time (about $10 \mathrm{~s}$ [3]). One can therefore consider the problem quasi-steady, the temperature $\mathrm{T}$ satisfying

$$
\frac{\mathrm{d}^{2} \mathrm{~T}}{\mathrm{dy}^{2}}=0
$$

here the $y$ - coordinate is measured across the film (see Fig. 2).

One of the most important observations of [3] is the fact that the mesoscopic structures were obtained only for very thin substrate thicknesses when the substrate was in the thermal equilibrium with the surrounding gas. We will therefore assume that the substrate temperature is constant and equal to $T_{\infty}$. We also assume equilibrium evaporation so that the film surface temperature is constant and equal to its saturation value $\mathrm{T}_{\mathrm{s}}$. Thus the boundary conditions for (1) are

$$
\left.\mathrm{T}\right|_{\mathrm{y}=0}=\mathrm{T}_{\infty} ;\left.\mathrm{T}\right|_{\mathrm{y}=\delta}=\mathrm{T}_{\mathrm{s}}
$$

The base problem (1)-(2) yields a linear temperature profile across the film

$$
\mathrm{T}=\mathrm{T}_{\infty}-\left(\mathrm{T}_{\infty}-\mathrm{T}_{\mathrm{s}}\right) \frac{\mathrm{y}}{\delta}
$$

Evaporation rate $\mathrm{J}$ is determined by the heat flux to the film surface

$$
\mathrm{JL}=-\left.\mathrm{k} \frac{\mathrm{dT}}{\mathrm{dy}}\right|_{\mathrm{y}=\delta}+\mathrm{h}\left(\mathrm{T}_{\infty}-\mathrm{T}_{\mathrm{s}}\right)
$$

where $\mathrm{k}$ is the heat conductivity and $\mathrm{L}$ is the latent heat of evaporation of the solvent.

Substituting (3) into (4), one obtains

$$
\mathrm{J}=\left(1+\frac{\mathrm{h} \delta}{\mathrm{k}}\right) \frac{\mathrm{k}\left(\mathrm{T}_{\infty}-\mathrm{T}_{\mathrm{s}}\right)}{\mathrm{L} \delta}
$$

then the equation for the film thickness will be

$$
\frac{\mathrm{d} \delta}{\mathrm{dt}}=-\frac{\mathrm{J}}{\rho}=-\left(1+\frac{\mathrm{h} \delta}{\mathrm{k}}\right) \frac{\mathrm{k}\left(\mathrm{T}_{\infty}-\mathrm{T}_{\mathrm{s}}\right)}{\mathrm{L} \delta \rho}
$$

here $\rho$ is the solution density.

The film thickness being small, the expression $h \delta / \mathrm{k}$ in (6) is negligible. Indeed, the typical value of $\mathrm{k}$ is $0.15 \mathrm{~W} /(\mathrm{mK})$ (see Ref. [10], Table 17.1). To make a rough estimation of $\mathrm{h}$ consider an impinging jet from a single slot nozzle. For the nozzle width of $1 \mathrm{~cm}$ and the air velocity of $3 \mathrm{~m} / \mathrm{s}$ (as in [3]) the recommended correlation [11, p. 432] predicts $\mathrm{h} \sim 25 \mathrm{~W} /\left(\mathrm{m}^{2} \mathrm{~K}\right)$ which yields for a $50 \mu \mathrm{m}$ film $\mathrm{h} \delta / \mathrm{k} \approx 8.3 \cdot 10^{-3}$. Neglecting this quantity, we can re-write (6) as

which yields

$$
\delta \frac{\mathrm{d} \delta}{\mathrm{dt}}=-\frac{\mathrm{k}\left(\mathrm{T}_{\infty}-\mathrm{T}_{\mathrm{s}}\right)}{\mathrm{L} \rho}=\mathrm{const}
$$

$$
\delta=\sqrt{\delta_{0}^{2}-\frac{2 \mathrm{k}\left(\mathrm{T}_{\infty}-\mathrm{T}_{\mathrm{s}}\right) \mathrm{t}}{\mathrm{L} \rho}}
$$

It can be shown [9] that solution (9) is the leading-order term in asymptotic 
expansion for slow evaporation.

Equation (8) predicts that the rate of thickness decrease is inversely proportional to the film thickness. Hence one would expect that perturbation of the film will cause higher rate of decrease in troughs and lower rate of decrease in crests resulting in growing perturbation and instability. To prove this, we will now analyze the perturbed solution.

\section{Perturbed solution}

We now impose a perturbation $\delta^{\prime}(\mathrm{x}, \mathrm{t})$ on the film thickness. The temperature field $\mathrm{T}(\mathrm{x}, \mathrm{y}, \mathrm{t})$ now satisfies

$$
\begin{aligned}
\frac{\partial \mathrm{T}}{\partial \mathrm{t}} & =\alpha\left(\frac{\partial^{2} \mathrm{~T}}{\partial \mathrm{x}^{2}}+\frac{\partial^{2} \mathrm{~T}}{\partial \mathrm{y}^{2}}\right) \\
\left.\mathrm{T}\right|_{\mathrm{y}=0} & =\mathrm{T}_{\infty} ;\left.\mathrm{T}\right|_{\mathrm{y}=\delta+\delta^{\prime}}=\mathrm{T}_{\mathrm{s}}
\end{aligned}
$$

while the evaporation rate will be determined from

$$
\mathrm{JL}=-\left.\mathrm{k} \frac{\partial \mathrm{T}}{\partial \mathrm{n}}\right|_{\mathrm{y}=\delta+\delta^{\prime}}+\mathrm{h}\left(\mathrm{T}_{\infty}-\mathrm{T}_{\mathrm{s}}\right)
$$

here the normal derivative to the film surface

$$
\frac{\partial \mathrm{T}}{\partial \mathrm{n}}=\frac{-\frac{\partial \mathrm{T}}{\partial \mathrm{x}} \frac{\mathrm{d} \delta^{\prime}}{\mathrm{dx}}+\frac{\partial \mathrm{T}}{\partial \mathrm{y}}}{\sqrt{1+\left(\frac{\mathrm{d} \delta^{\prime}}{\mathrm{dx}}\right)^{2}}}
$$

Writing the temperature as the sum of the base value $\bar{T}(\mathrm{y}, \mathrm{t})$ and the perturbation $\mathrm{T}^{\prime}(\mathrm{x}, \mathrm{y}, \mathrm{t})$ and linearizing, one obtains

$$
\begin{gathered}
\frac{\partial \mathrm{T}}{\partial \mathrm{n}} \approx-\frac{\partial \mathrm{T}}{\partial \mathrm{x}} \frac{\mathrm{d} \delta^{\prime}}{\mathrm{dx}}+\frac{\partial \mathrm{T}}{\partial \mathrm{y}}=\frac{\partial \mathrm{T}}{\partial \mathrm{y}} \\
\left.\left.\frac{\partial \mathrm{T}}{\partial \mathrm{y}}\right|_{\mathrm{y}=\delta+\delta^{\prime}} \approx \frac{\partial \mathrm{T}}{\partial \mathrm{y}}\right|_{\mathrm{y}=\delta}+\left.\delta^{\prime} \frac{\partial^{2} \overline{\mathrm{T}}}{\partial \mathrm{y}^{2}}\right|_{\mathrm{y}=\delta}=\left.\frac{\partial \mathrm{T}}{\partial \mathrm{y}}\right|_{\mathrm{y}=\delta} \\
\left.\left.\mathrm{T}\right|_{\mathrm{y}=\delta+\delta^{\prime}} \approx \mathrm{T}\right|_{\mathrm{y}=\delta}+\left.\delta^{\prime} \frac{\partial \overline{\mathrm{T}}}{\partial \mathrm{y}}\right|_{\mathrm{y}=\delta}=\left.\mathrm{T}\right|_{\mathrm{y}=\delta}-\left(\mathrm{T}_{\infty}-\mathrm{T}_{\mathrm{s}}\right) \frac{\delta^{\prime}}{\delta}
\end{gathered}
$$

Then, instead of (10)-(12) we can write

$$
\begin{gathered}
\frac{\partial \mathrm{T}^{\prime}}{\partial \mathrm{t}}=\alpha\left(\frac{\partial^{2} \mathrm{~T}^{\prime}}{\partial \mathrm{x}^{2}}+\frac{\partial^{2} \mathrm{~T}^{\prime}}{\partial \mathrm{y}^{2}}\right) \\
\left.\mathrm{T}^{\prime}\right|_{\mathrm{y}=0}=0 ;\left.\mathrm{T}^{\prime}\right|_{\mathrm{y}=\delta}=\left(\mathrm{T}_{\infty}-\mathrm{T}_{\mathrm{s}}\right) \frac{\delta^{\prime}}{\delta} \\
\mathrm{JL}=-\left.\mathrm{k} \frac{\partial \overline{\mathrm{T}}}{\partial \mathrm{y}}\right|_{\mathrm{y}=\delta}-\left.\mathrm{k} \frac{\partial \mathrm{T}^{\prime}}{\partial \mathrm{y}}\right|_{\mathrm{y}=\delta}+\mathrm{h}\left(\mathrm{T}_{\infty}-\mathrm{T}_{\mathrm{s}}\right)
\end{gathered}
$$

It follows from (19) and (4) that the evaporation rate perturbation $\mathrm{J}^{\prime}$ is

$$
\mathrm{J}^{\prime}=-\left.\frac{\mathrm{k}}{\mathrm{L}} \frac{\partial \mathrm{T}^{\prime}}{\partial \mathrm{y}}\right|_{\mathrm{y}=\delta}
$$

and therefore 


$$
\frac{\mathrm{d} \delta^{\prime}}{\mathrm{dt}}=-\frac{\mathrm{J}^{\prime}}{\rho}=\left.\frac{\mathrm{k}}{\mathrm{L} \rho} \frac{\partial \mathrm{T}^{\prime}}{\partial \mathrm{y}}\right|_{\mathrm{y}=\delta}
$$

Defining dimensionless temperature perturbation,

$$
\theta^{\prime}=\frac{\mathrm{T}^{\prime}}{\mathrm{T}_{\infty}-\mathrm{T}_{\mathrm{s}}}
$$

we re-write (17)-(18) as

$$
\begin{aligned}
& \frac{\partial \theta^{\prime}}{\partial \mathrm{t}}=\alpha\left(\frac{\partial^{2} \theta^{\prime}}{\partial \mathrm{x}^{2}}+\frac{\partial^{2} \theta^{\prime}}{\partial \mathrm{y}^{2}}\right) \\
& \left.\theta^{\prime}\right|_{\mathrm{y}=0}=0 ;\left.\theta^{\prime}\right|_{\mathrm{y}=\delta}=\frac{\delta^{\prime}}{\delta}
\end{aligned}
$$

Since the perturbation can change with time much faster then the base state, one cannot a priory consider the perturbed problem quasi-steady. However, such an assumption can be made after appropriate change of coordinates. We define

$$
\mathrm{s}=\frac{\mathrm{x}}{\delta}, \mathrm{z}=\frac{\mathrm{y}}{\delta}, \tau=\ln \frac{\delta_{0}}{\delta}
$$

in the new coordinates, $(23)$ becomes

$$
\frac{\partial^{2} \theta^{\prime}}{\partial \mathrm{s}^{2}}+\frac{\partial^{2} \theta^{\prime}}{\partial \mathrm{z}^{2}}=-\frac{\delta}{\alpha} \frac{\mathrm{d} \delta}{\mathrm{dt}}\left(\frac{\partial \theta^{\prime}}{\partial \tau}+\mathrm{z} \frac{\partial \theta^{\prime}}{\partial \mathrm{z}}+\mathrm{s} \frac{\partial \theta^{\prime}}{\partial \mathrm{s}}\right)
$$

or on substituting the thickness derivative from (8)

$$
\frac{\partial^{2} \theta^{\prime}}{\partial \mathrm{s}^{2}}+\frac{\partial^{2} \theta^{\prime}}{\partial \mathrm{z}^{2}}=\mathrm{K}\left(\frac{\partial \theta^{\prime}}{\partial \tau}+\mathrm{z} \frac{\partial \theta^{\prime}}{\partial \mathrm{z}}+\mathrm{s} \frac{\partial \theta^{\prime}}{\partial \mathrm{s}}\right)
$$

where $K=\frac{c_{p}\left(T_{\infty}-T_{s}\right)}{L}$ and $c_{p}$ is the specific heat of the polymer solution.

The parameter $\mathrm{K}$ is called Kutateladze number [7,8] or Jakob number [12]. Its value is usually very low due to the high value of heat of evaporation and small temperature difference across the thin film. For example, in both [7] and [8] it was taken as 0.01 . For the solvents used in [3] the value of $\mathrm{K}$ would be less than 0.05 even for the temperature difference of $10^{\circ}$ across the film (see Table 1).

We will therefore neglect the right-hand side of (27) to obtain the following perturbed problem in the transformed coordinates

$$
\begin{gathered}
\frac{\partial^{2} \theta^{\prime}}{\partial \mathrm{s}^{2}}+\frac{\partial^{2} \theta^{\prime}}{\partial \mathrm{z}^{2}}=0 \\
\left.\theta^{\prime}\right|_{\mathrm{z}=0}=0 ;\left.\theta^{\prime}\right|_{\mathrm{z}=1}=\frac{\delta^{\prime}}{\delta_{0}} \mathrm{e}^{\tau}
\end{gathered}
$$

The second condition in (29) follows from the definition of $\tau(25)$.

The normal-mode analysis can be applied to (28)-(29). We write

$$
\begin{aligned}
& \theta^{\prime}=\hat{\theta}(\mathrm{z}, \tau) \mathrm{e}^{\mathrm{i} \kappa \mathrm{s}} \\
& \frac{\delta^{\prime}}{\delta_{0}}=\mathrm{A}(\tau) \mathrm{e}^{\mathrm{i} \kappa \mathrm{s}}
\end{aligned}
$$

to obtain

$$
\begin{gathered}
\frac{\mathrm{d}^{2} \hat{\theta}}{\mathrm{dz}^{2}}-\kappa^{2} \hat{\theta}=0 \\
\left.\hat{\theta}\right|_{\mathrm{z}=0}=0 ;\left.\hat{\theta}\right|_{\mathrm{z}=1}=\mathrm{A}(\tau) \mathrm{e}^{\tau}
\end{gathered}
$$


with the solution

$$
\hat{\theta}=\mathrm{A}(\tau) \mathrm{e}^{\tau} \frac{\sinh \kappa z}{\sinh \kappa}
$$

Substituting (34) into (21) and using the new coordinates, one obtains

$$
\frac{\mathrm{d} \delta^{\prime}}{\mathrm{dt}}=\frac{\mathrm{k}\left(\mathrm{T}_{\infty}-\mathrm{T}_{\mathrm{s}}\right)}{\mathrm{L} \rho} \frac{\mathrm{A}(\tau) \mathrm{e}^{\tau}}{\delta} \frac{\kappa}{\tanh \kappa} \mathrm{e}^{\mathrm{i} \kappa \mathrm{s}}
$$

on the other hand,

$$
\frac{\mathrm{d} \delta^{\prime}}{\mathrm{dt}}=-\frac{1}{\delta} \frac{\mathrm{d} \delta}{\mathrm{dt}} \frac{\mathrm{d} \delta^{\prime}}{\mathrm{d} \tau}=\frac{\mathrm{k}\left(\mathrm{T}_{\infty}-\mathrm{T}_{\mathrm{s}}\right)}{\mathrm{L} \rho \delta^{2}} \frac{\mathrm{d} \delta^{\prime}}{\mathrm{d} \tau}
$$

Substituting (35) into (36) and using (8), (31) yields

$$
\frac{\mathrm{dA}}{\mathrm{d} \tau}=\mathrm{A}(\tau) \frac{\delta \mathrm{e}^{\tau}}{\delta_{0}} \frac{\kappa}{\tanh \kappa}=\mathrm{A}(\tau) \frac{\kappa}{\tanh \kappa}
$$

Therefore, the solution for the perturbation amplitude is

$$
\mathrm{A}(\tau)=\mathrm{A}(0) \exp \left(\frac{\kappa \tau}{\tanh \kappa}\right)
$$

One sees from (38) that the perturbation grows for all wave numbers and the base state is unstable.

\section{Discussion}

We have obtained that evaporation of a thin polymer film is unstable. The instability found is thermal and caused by increasing of evaporation with the film thinning due to higher heat flux to the film surface. It can be responsible for the creation of mesoscopic structures in the experiments of Bormashenko et al. [3].

It is seen from (38) the perturbation growth increases with the wave number. However, for sufficiently large $\kappa$ the derivative $\partial \mathrm{T} / \partial \mathrm{x}$ becomes too large and neglecting the inertial terms is no longer justified. Only the full analysis that includes both thermal, flow and diffusion problem can find the most unstable wave number.

\section{REFERENCES}

1. T.Liu, C. Burger and B. Chu (2003) Nanofabrication in polymer matrices. Prog. Polymer Sci., Vol. 28, No. 1, pp. 5-26.

2. S. J. Teertstra and M. Gauthier (2004) Dendrigraft polymers: macromolecular engineering on a mesoscopic scale. Prog. Polymer Sci., Vol. 29, No. 4, pp. 277-327

3. E. Bormashenko, R. Pogreb, O. Stanevsky, E. Bormashenko, T. Stein, V.-Z. Gaisin, R. Cohen and O. V. Gendelman (2005) Mesoscopic pattering in thin polymer films formed under the fast dip-coating process. Macromol. Mater. Eng., Vol. 290, pp. 114-121.

4. L. Weh (2001) Hyperbolic spirals as surface structures in thin layers. $J$. Colloid Interface Sci., Vol. 235, No. 2, pp. 210-217.

5. P.G. de Gennes (2001) Instabilities during the evaporation of a film: Nonglassy polymer + volatile solvent. Eur. Phys. J. E, Vol. 6, No. 5, pp. 421-424.

6. A. Oron, S. H. Davis, and S. G. Bankoff (1997) Long-scale evolution of thin liquid films. Rev. Mod. Phys., Vol. 69, No. 3, pp. 931-980.

7. B. Spindler (1982) Linear stability of liquid films with interfacial phase change. Int. J. Heat Mass Transfer, Vol. 25, No. 2, pp. 161-173. 
8. S. P. Aktershev and S. V. Alekseenko (2005) Influence of condensation on the stability of a liquid film moving under the effect of gravity and turbulent vapor flow. Int. J. Heat Mass Transfer, Vol. 48, No. 6, pp. 1039-1052.

9. J. P. Burelbach, S. G. Bankoff and S. H. Davis (1988) Nonlinear stability of evaporating/condensing liquid films. J. Fluid Mech., Vol. 195, pp. 463-494.

10. D. W. Van Krevelen and P. J. Hoftyzer (1976) Properties of Polymers, $2^{\text {nd }}$ ed., Elsevier.

11. F. P. Incropera and D. P. Dewitt (2002) Fundamentals of Heat and Mass Transfer, $5^{\text {th }}$ ed., Wiley.

12. C. T. Avedisian (1985) Bubble growth in superheated liquid droplets. In: N. P. Cheremisinoff (ed.), Encyclopedia of Fluid Mechanics, Gulf, Vol. 3, pp. 130190.

13. The data courtesy of NIST Chemistry WebBook http://webbook.nist.gov/chemistry.

Table 1: Properties of the solvents [13].

\begin{tabular}{|l|c|c|c||}
\hline \multirow{2}{*}{ Solvent } & $\mathrm{L}$ & $\mathrm{c}_{\mathrm{p}}$ & $\mathrm{c}_{\mathrm{p}} / \mathrm{L}$ \\
\cline { 2 - 4 } & $\mathrm{kJ} / \mathrm{mol}$ & $\mathrm{J} / \mathrm{mol} \cdot \mathrm{K}$ & $1 / \mathrm{K}$ \\
\hline \hline Dichloromethane & 28.06 & 102.3 & $3.65 \cdot 10^{-3}$ \\
\hline Chloroform & 29.24 & 114.25 & $3.91 \cdot 10^{-3}$ \\
\hline
\end{tabular}

\section{List of Figures}

Fig. 1: Schematics of the dip-coating process [3].

Fig. 2: Simplified model of the polymer film evaporation. 


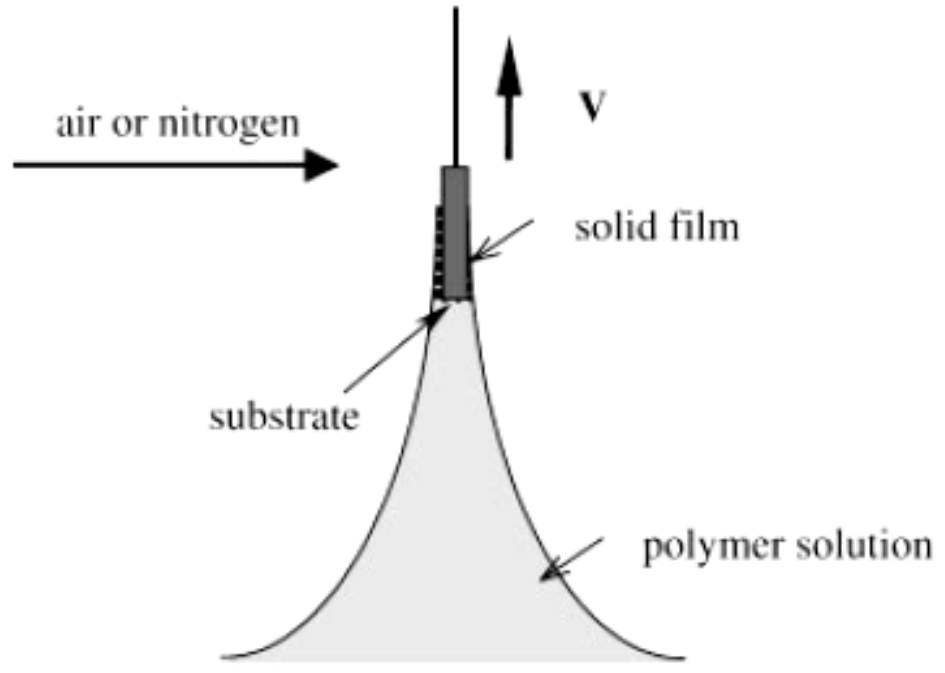

Fig. 1: Schematics of the dip-coating process [3].

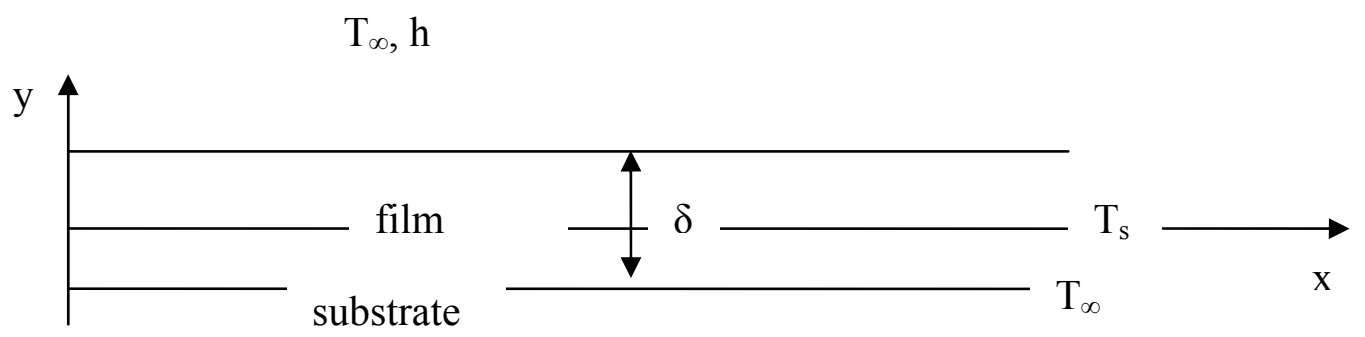

Fig. 2: Simplified model of the polymer film evaporation 